\title{
Modelling of surface identifying characteristics using Fourier series
}

Conference or Workshop Item

Accepted Version

Wall, S. S. and Harwin, W. S. (1999) Modelling of surface identifying characteristics using Fourier series. In: The Winter Annual Meeting of ASMEDSC - ASME Dynamic Systems and Control Division, 1999, pp. 65-71. Available at http://centaur.reading.ac.uk/19315/

It is advisable to refer to the publisher's version if you intend to cite from the work. See Guidance on citing.

All outputs in CentAUR are protected by Intellectual Property Rights law, including copyright law. Copyright and IPR is retained by the creators or other copyright holders. Terms and conditions for use of this material are defined in the End User Agreement. 


\section{CentAUR}

Central Archive at the University of Reading

Reading's research outputs online 


\title{
DSC-2-4
}

\section{MODELLING OF SURFACE IDENTIFYING CHARACTERISTICS USING FOURIER SERIES.}

\author{
Steven A. Wall and William S. Harwin \\ The Department of Cybernetics \\ University of Reading \\ Whiteknights \\ PO Box 225 \\ Reading RG6 6AY \\ UK \\ Facsimile: +44 (0) 1189318220 \\ E-mail: saw@cyber.rdg.ac.uk, w.s.harwin@reading.ac.uk www: http://www.rdg.ac.uk/ shr97saw
}

\begin{abstract}
Texture and small-scale surface details are widely recognised as playing an important role in the haptic identification of objects. In order to simulate realistic textures in haptic virtual environments, it has become increasingly necessary to identify a robust technique for modelling of surface profiles. This paper describes a method whereby Fourier series spectral analysis is employed in order to describe the measured surface profiles of several characteristic surfaces. The results presented suggest that a bandlimited Fourier series can be used to provide a realistic approximation to surface amplitude profiles.
\end{abstract}

\section{INTRODUCTION}

\subsection{Object Surface Properties}

As with temporal signals, spatial information can be characterised by its amplitude and frequency. The large-scale geometric shape of an object can be deemed low frequency, high amplitude information. Smaller scale surface properties, such as the texture of the object are, conversely, low amplitude and high frequency. Indeed, qualitative classification of surface textures is often analogous to a spectral description of the surface properties. Typical adjectives chosen by subjects include rough, smooth, coarse, fine, granulated, rippled, regular and irregular [1]. Some denote the periodicity of the surface, some the amplitude of the features and others indicate a bandwidth of frequency components. This implies that through modification of a spatial power spectrum, the subject's perception as to the nature of the surface being displayed can be altered.

Katz [2] defined two types of surface properties. Qualities are properties on which any surface can be rated, for example, roughness. Identifying characteristics are the overall tactile impressions of the surface, for example, the 'rubberiness' of rubber. Katz postulated that identifying characteristics may be the result of certain combinations or values of qualities. If this was not the case, it was some undefinable material quality which gave a surface its characteristic feel, and two surfaces with the same combinations of qualities would not necessarily be perceived as identical under haptic exploration. Katz did not offer a solution to the problem, but his work prompted the more general premise that perceptions of surface texture may be interrelated in such a fashion that they can be expressed by a perceptual space. Results demonstrated that a subject's judgement of surface texture can be represented in a perceptual space of three or more dimensions, though descriptors remained elusive. It is likely, however, that 'rough-smooth' and 'hard-soft' scales comprise two such perceptual dimensions. Regardless, the results did imply that identifying characteristics were dependent upon the values of qualities [3]. Thus, it is plausible to infer further, that by providing a tactile representation derived from spatial descriptions relevant to such qualities, it is possible to evoke a perceptual response comparable to those produced by real world surfaces.

\subsection{Merits of Texture Display}

When exploring a surface with a probe, for example, writing on paper using a pen, it is possible for the user to make judgements on the material properties of the surface via high frequency vibrations transmitted through the probe $[2,4]$. Thus, the provision of vibratory information allows the assessment of the texture of a surface.

When large scale shape information is limited, texture becomes a defining factor in the identification of objects, particularly when texture is stereotypical of the object being identified. Identification of objects during spatially constrained 
contact (a haptic glance) demonstrated results well above chance, indicating that it is possible to build a volumetric model from sparse spatial data, and the basis for this recognition is other than large scale structure, thus illustrating the importance of material information [5].

Haptic performance is most likely to benefit from material information when structural information is limited [6]. As many haptic interfaces employ a single fingertip interface, the addition of material information transmitted via vibration is likely to have a substantial effect.

The addition of high frequency information has been shown to improve performance in HCI. Typically, response times and accuracy are improved when force feedback is augmented by tactile displays [7]. Providing a mouse with tactile feedback in the form of a solenoid driven pin raised against the user's fingerpad reduced target selection times, though the speed advantage was obviated by a decrease in user accuracy [10].

\subsection{Previous Developments in Tactile Display}

Having established that provision for vibratory information is beneficial to performance, it is then necessary to establish a robust algorithm for subsequent display of haptic phenomena.

The Sandpaper system, developed by Minsky et al [8], utilised a 2 degrees of freedom (DOF) joystick that recreated surface textures using virtual springs which pull the users hand towards low regions and away from high regions of a texture depth map. Materials were specified in terms of density and placement of springs rather than material qualities or identifying characteristics. As no force was present normal to the contact surface, the sandpaper system demonstrates that tangential forces are capable of representing surface qualities.

Conversely, texture display was implemented using the PHANToM (SensAble technologies, Cambridge, MA) by employing a virtual surface defined by a sinusoid [9]. The software performed collision checks with a planar surface that was perturbed in a direction normal to contact by a sinusoid, in accordance with the user's position. Thus, it can be concluded that either normal or tangential forces are sufficient for texture display.

Akamatsu et al[10] simulated virtual textures with a mouse adapted for tactile display, by raising a solenoid driven pin against the users finger. Information was gathered from live surfaces using an apparatus with an accelerometer and strain gauges in order to measure vibration and friction produced by touching a real texture with the fingertip. The velocity of exploration and force of contact were controlled such that a material was moved along the fingertip with the same velocity and force irrespective of the material. The vibration patterns induced suggested that amplitude, pitch and irregularity of these factors can be parameters of virtual texture. It was found that subjects could reliably discriminate between textures presented in this fashion, though there was no indication as to how well the psychological impression produced by the models mimicked there real world counterparts.

Similarly, Okamura et al [11] gathered a library of real world vibration parameters by collecting and modelling data obtained during task execution. A stylus mounted with an accelerometer was stroked across a surface in order to gather vibratory information regarding the surface properties. Vibrations could then be replayed through the device in order to recreate the sensation of texture. Characteristics chosen to describe texture were mean and maximum power frequency from a power spectral analysis, and the rms. value of the vibration signal. It was found that frequency parameters did not depend on the velocity of the stylus, but amplitude was dependent on applied force and velocity. The vibration was applied in the direction of motion, parallel to the virtual surface. The results obtained indicated a realistic representation of surface identifying characteristics can be obtained using a deterministic model with several parameters.

Basdogan et al [12] described several methods pertaining to haptic texture synthesis. Image based haptic texturing involves constructing a texture field from $2 \mathrm{D}$ image data. Thus, the greyscale intensity of the image data corresponds to a height indicator in the haptic simulation. A drawback of this system is that it is dependant on the availability of the image data, which is also costly in terms of required storage space, particularly where many surfaces are to be simulated. The second method describes a procedural technique, whereby Fourier series and stochastic functions, or a combination of these two, could be used to generate haptic textures. It was noted that several different textures could be generated by modifying coefficients of a Fourier series. Fractal methods were also considered, using a Fourier series as the basis function, then recursively subdividing height and frequency descriptors until the desired level of detail was obtained. This level is established by the spatial and temporal resolution of the human and the haptic device.

Green and Salisbury [13] outlined a 3-step process to texture simulation with a PHANToM. Data was gathered using a hybrid control scheme that allowed the distal point of the PHANToM to be stroked across a test surface at a constant velocity. Positional data was then related to lateral and normal forces during exploration, which was then replayed to the user. It was noted that the elements of the force vector fell in to a Gaussian distribution.

All methods considered so far have adopted a deterministic approach to texture representation. Siira and Pai [14] used a stochastic method in which textures were approximated by a Gaussian distribution, the parameters of which were dependant upon measured surface properties. The Gaussian approach was deemed sufficient, given that computational overheads restrict accurate representation of texture in real time applications. Also, given the limits of human tactile capabilities, a realistic approximation of surface texture may produce the desired psychophysical impression. A virtual surface was implemented combining normal constraint forces and texture impulses with tangential texture impulses, and it was also noted that forces proportional to the normal constraint force created a more realistic feeling texture.

Fritz and Barner[1] reproduced Gaussian texture effects in 3D using a PHANToM. Their aim, however, was not to simulate real textures, but to produce textures which were perceptually different. It was found that simple textures could be produced from a multivariate probability density function (PDF, e.g. uniform, Gaussian), and increasingly complex surfaces could be portrayed using a combination of a number of Gaussian PDFs. Textures produced by a Gaussian noise primitive were rough, like 'granite or gravel', higher variance producing a rougher texture.

\subsection{High Frequency Probe Augmented PHANToM}

Force feedback type interfaces are typically limited to the display of low frequency, high amplitude spatial properties. Due 
to the mechanical framework inherent in the design of such devices, high frequency information tends to be severely attenuated, though some crude cutaneous sensation can be presented to the user. In order to display accurate information regarding small-scale surface properties, it is necessary to augment an existing device with an actuator for tactile display, which is capable of providing the necessary cutaneous information.

Basdogan et al [12] noted that the resolution of the PHANToM does not match that of the human finger, thus, modification of surface contact forces was required prior to display. Moreover, the level of detail attainable in haptic rendering is set by the spatial and temporal resolution of the haptic display employed. The method adopted in the subsequent work utilises a PHANToM, augmented with a pen-like vibrotactile end effector based upon a voice coil motor, as illustrated in Figure 1.[15]. The device is gripped between the users thumb and finger, hence maximising contact area and, thus, sensitivity to mechanical vibrations induced in the probe [16]. However, when exploring a surface via remote contact using a probe, there is no provision for spatially intensive representation of surface geometry, as with direct contact. The user is instead restricted to temporally varying vibration cues related to the distal geometry of the stimulus surface. As such, performance in discriminatory tasks is degraded, though assessment of material qualities, such as roughness, is still possible and effective. Accuracy in identifying common objects was poorer than for direct contact, due to lack of large-scale geometric cues. [17].

\section{MODELLING OF SURFACE IDENTIFYING CHARACTERISTICS BY FOURIER SERIES}

It has been noted that object surface patterns often can be characterised by roughly repeatable patterns. It is desired to develop a procedural technique for representing surface qualities. Thus, different textures could be created through variation of a few parameters that require little storage space. It has already been established that a subject's qualitative description of surface characteristics is analogous to parameters of a spectral description. Some success has also been achieved in the field of texture modelling using power spectral descriptions. It was therefore deemed appropriate to pursue further investigation into the representation of surface profiles using Fourier series, as a basis towards establishing a procedural technique.

\subsection{Data Collection}

In order to model surface texture it was first necessary to gather a measure of the small-scale geometric surface profile. The high frequency probe used to display such data provided a

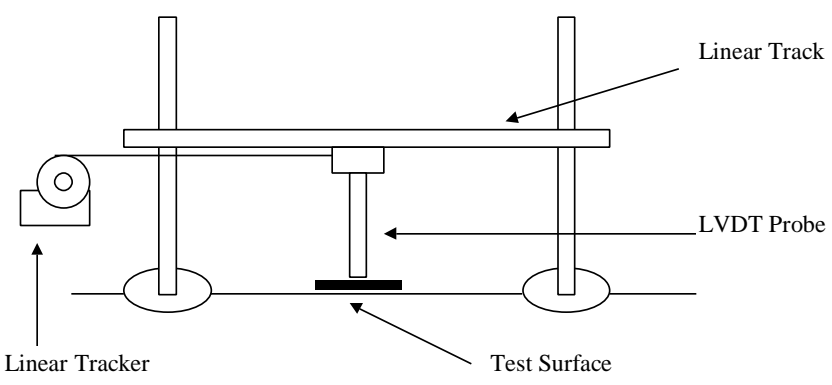

Figure 2: Apparatus for gathering test surface data.

convenient means of gathering the amplitude of surface profiles. The probe was suspended from a linear shaft at a roughly constant height above the surface to be measured. The position of the probe along the shaft could be measured by a linear tracking device. The tracking device consisted of a rotary encoder and capstan, with a sensitivity of $0.0049 \mathrm{~V} / \mathrm{mm}$. Data was interpreted by a CIODAS1602/12 (Computer Boards Inc., Mansfield, MA) interface board, with a resolution of 0.00244 volts, giving a corresponding positional resolution of $0.49 \mathrm{~mm}$. In order to gather texture data, the probe was stroked across the test surface. The experimental set-up is illustrated in figure 2. Some effort was made to maintain a constant velocity of exploration, though this condition was not strictly enforced, as subsequent processing of the data rendered such a precaution unnecessary. Readings from the probe were taken at a sampling period of $0.01 \mathrm{~s}$. The spatial resolution of the readings is dependent on the translational velocity of the probe during exploration of the test surfaces. The mean velocity recorded over all test surfaces was $22.5 \mathrm{~mm} / \mathrm{s}$, with a standard deviation of $8.2 \mathrm{~mm} / \mathrm{s}$. The worst case scenario for sampling occurs at the highest translational velocity, which was recorded as $36.95 \mathrm{~mm} / \mathrm{s}$. Given the temporal sampling period, this corresponds to spatial sampling period of $0.3695 \mathrm{~mm}$. Signals from the sensing element of the probe therefore recorded amplitude information regarding the small-scale surface details, along with a reference position from the linear tracker. The sensitivity of the LVDT used in the probe was $0.68 \mathrm{~V} / \mathrm{mm}$, and is linear over a distance of $\pm 3 \mathrm{~mm}$, which is sufficient for the detection of surface geometry. The resolution of the LVDT is limited by the method by which the voltages are interpreted, for example, an A/D converter used to read into a controlling computer. In this case, the CIODAS1602/12 (Computer Boards Inc., Mansfield, MA) has a resolution of 0.00244 Volts, corresponding to a positional resolution of $0.0035 \mathrm{~mm}$. Data was gathered for five surface types: corrugated cardboard, mouse mat fabric, sandpaper, wood with movement along the grain, and wood with movement against

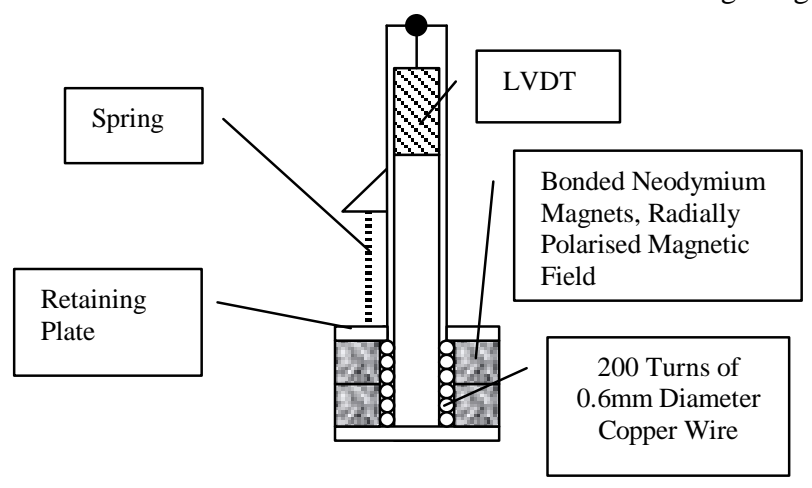

Figure 1: Design of high frequency probe actuator. 


\section{Sandpaper}

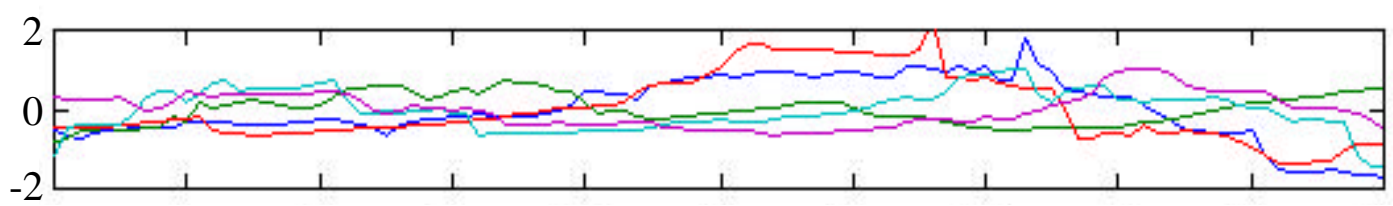

Cardboard
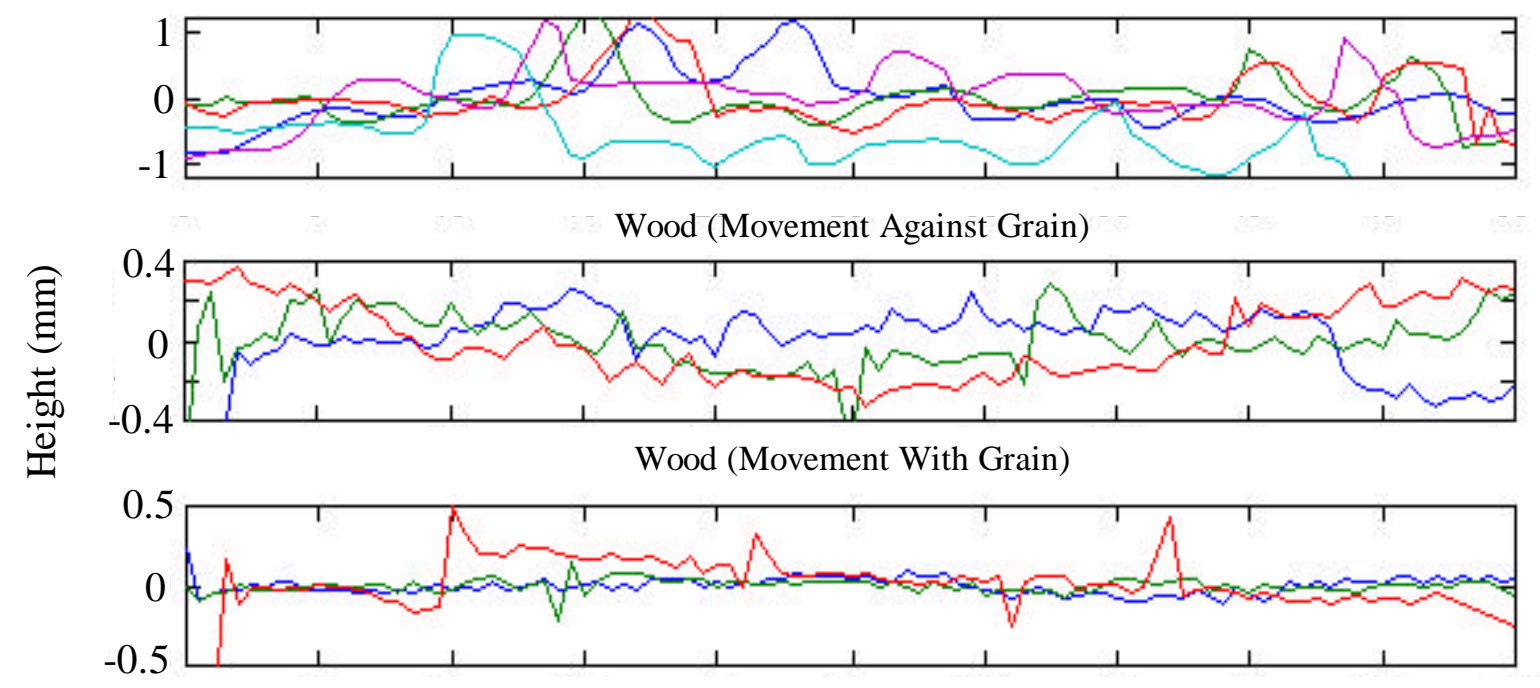

Mouse mat

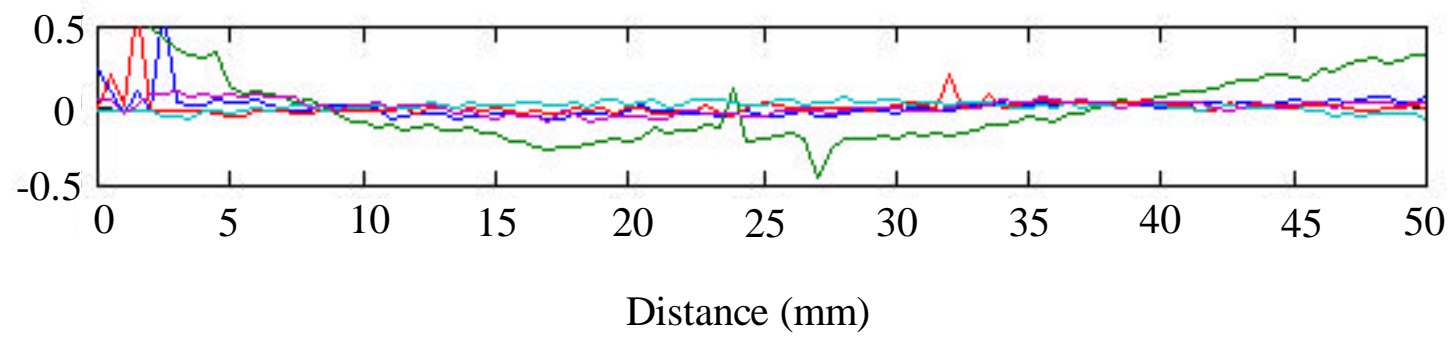

Figure 3. Surface profile measurements for all surfaces.

\subsection{Processing and Interpretation of Data}

The translational position and height data was combined to provide a series of two-dimensional surface profiles. The first step in the process of constructing such a surface description was to filter the translational position measure, to eliminate the effects of high frequency noise. As the rate of change of horizontal position was roughly constant, a simple method to filter the data was resampling every $0.1 \mathrm{~s}$ and linearly interpolating between the sample points. Data was then cross referenced with the corresponding index of positional height data to give a height reading, $y$, for each linear position, x. This data was then also linearly interpolated, providing a surface profile map equivalent to $50 \mathrm{~mm}$ of the material, at a spatial sampling interval of $0.5 \mathrm{~mm}$. The best straight line fit was then removed from the data, providing some protection from spurious results in the data arising from the non- probe was mounted, though further processing would likely eliminate such discrepancies. Surface profiles resulting from the above processing are illustrated in figure 3 .

Taking discrete Fourier transforms of each surface profile, several trends were apparent in the results. All surfaces exhibited a large peak in the amplitude spectra in the $2^{\text {nd }}$ harmonic, corresponding to a spatial frequency of 20 cycles / $\mathrm{m}$ (wavelength of $50 \mathrm{~mm}$ ). It was surmised that this was a result of residuals from the non-parallel nature of the surface and linear shaft, which the detrending of the data had failed to eliminate, due to its non-linear nature. It was also noted, as was predicted, that the surfaces generally perceived as 'rougher' had a larger magnitude of amplitude spectra, though psychophysical testing would likely yield more robust conclusions. 


\subsection{Synthesis of Textures}

It was observed that the amplitude spectra for each surface differed within the different samples for that particular surface, though they were generally of a similar order of magnitude. To account for such inter-variation within samples of the same surface, the basic template for a Fourier series of each surface was therefore chosen as the mean and standard deviation of the real and imaginary components of the amplitude spectra. Thus, 'new' textures for a given test material can be constructed by calculating the Fourier coefficients based upon a Gaussian distribution of given mean and standard deviation. Hence, surfaces can be expressed in terms of its composite harmonics as follows:

$f(x)=\frac{1}{101} \sum_{n=1}^{n=50} a_{n} \cos \left(n \omega_{0} x\right)+\frac{1}{101} \sum_{n=1}^{n=50} b_{n} \sin \left(n \omega_{0} x\right)$

where: $\quad f(x)=$ Surface profile description.

$\omega_{0}=$ Fundamental spatial frequency $=20$ cycles $/ \mathrm{m}$

$\mathrm{x}=$ Displacement $(\mathrm{m})$

$a_{n}, b_{n}=$ Gaussian white noise variables chosen from a PDF of known mean and variance.

Problems occurred initially with this method as it was noted during visual appraisal of data that low frequency data tended to characterise the results. Thus, the underlying high amplitude sinusoid previously discussed tended to appear in all subsequent models for the surface. This effect is illustrated for the mouse mat fabric data. Figure 4 shows the mean surface profile obtained from the test data, which clearly illustrates the presence of a dominant, low frequency harmonic of large amplitude. Figure 5 illustrates a surface created using the above method. It can be observed that the low frequency information is present in the 'new' surface.

In order to eliminate such spurious results, the spectra was band-limited to the range 100-1000 cycles / $\mathrm{m}$. The mean exploration velocity of 15 subjects recorded during haptic interaction was roughly $0.1 \mathrm{~m} / \mathrm{s}$. The bandwidth of the PHANToM

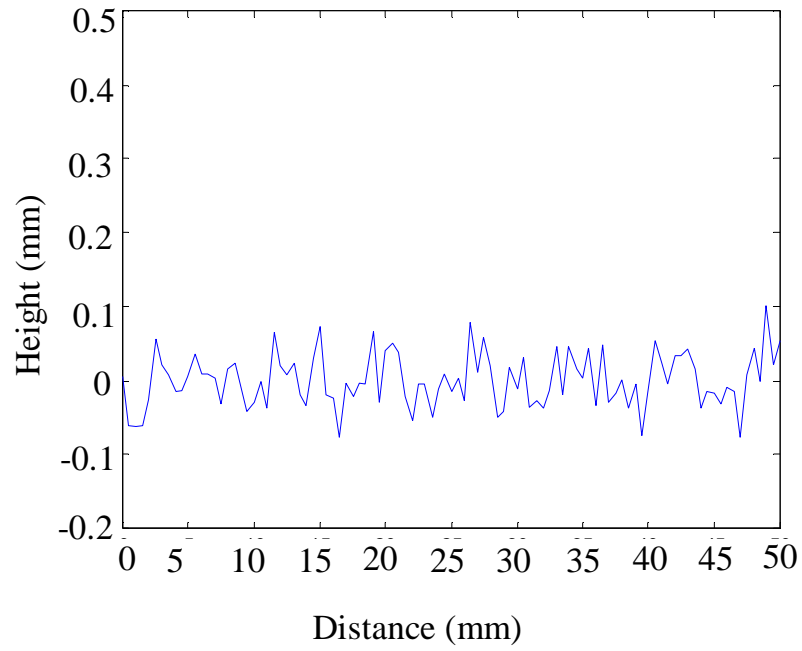

Figure 6: Mouse mat surface profile using bandlimited Fourier series.

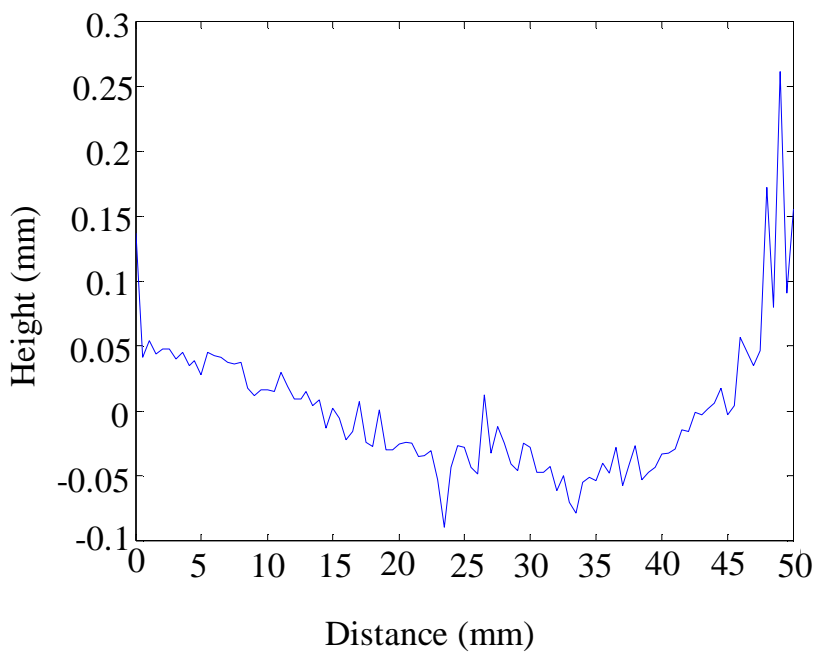

Figure 4: Mean surface profile for mouse mat.

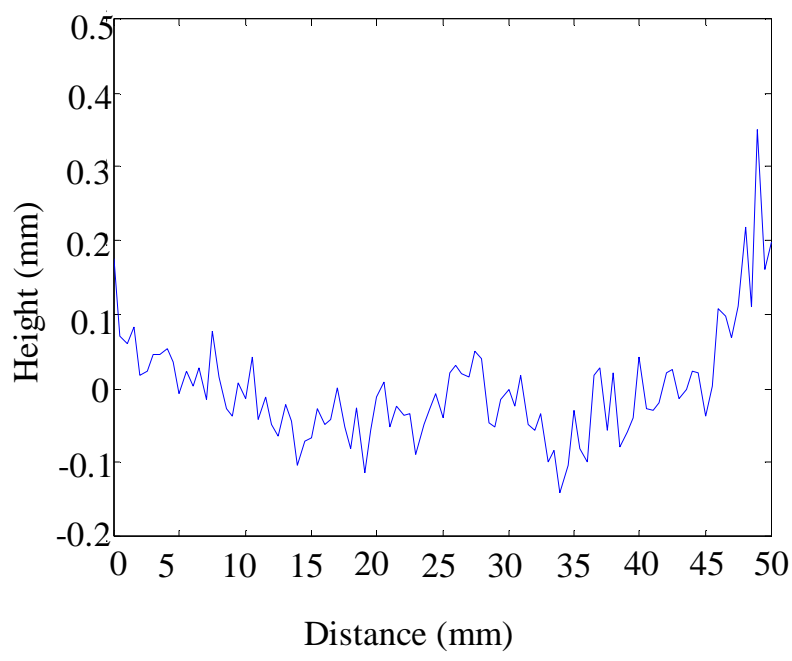

Figure 5: Recreated mouse mat surface profile.

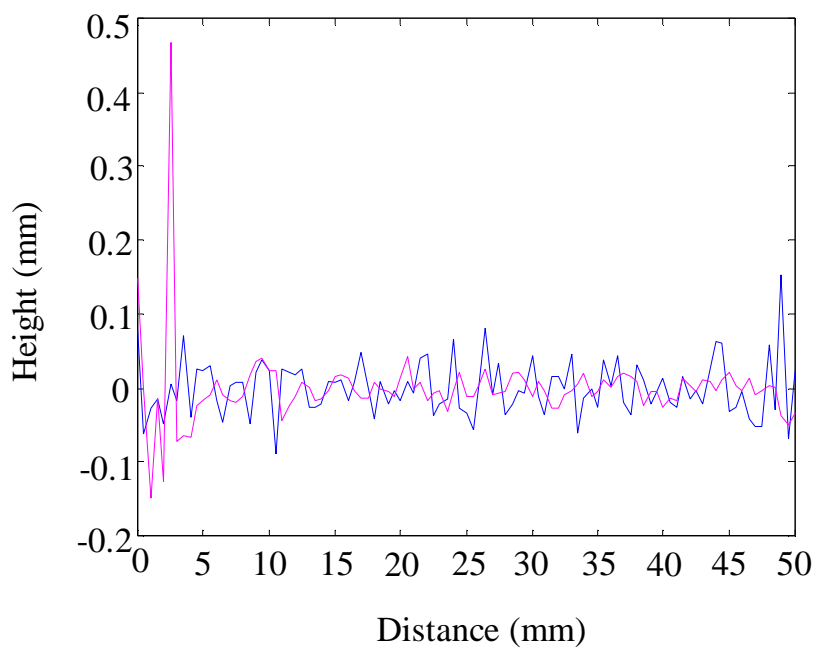

Figure 7: Comparison of simulated surface and original data after filtering. 
is $10 \mathrm{~Hz}$ [15], which, assuming average exploration velocity, corresponds to a spatial frequency of 100 cycles/m.. Hence, this was selected as the cut-off frequency, as the high frequency probe will be used to display surface characteristics the PHANToM is incapable of representing. Thus, the revised expression for surface profile representation is:

$f(x)=\frac{1}{101} \sum_{n=6}^{50} a_{n} \cos \left(n \omega_{0} x\right)+\frac{1}{101} \sum_{n=6}^{50} a_{n} \sin \left(n \omega_{0} x\right)$

Subsequent generation of sample textures confirmed that the anomalies had been removed, as illustrated in figure 6 . Figure 7 illustrates a comparison between a surface generated using the above method, and the original data gathered for the surface, after being bandlimited to the range 100-1000 cycles/m. .Visual analysis of the inverse Fourier transform of the mean Fourier series for each surface suggests that the data is in some fashion related to the surface qualities. For example, the cardboard mean surface profile exhibited a periodicity that could be attributed to the corrugated nature of the surface, while 'rougher' surfaces tended to have larger amplitude features. This can be expressed by the standard deviation of the mean surface profiles, shown in Table 1.

\begin{tabular}{|l|l|}
\hline Material & $\begin{array}{l}\text { Standard Deviation of Filtered } \\
\text { Mean Surfaces (mm) }\end{array}$ \\
\hline Wood (Against Grain) & 0.0207 \\
\hline Mouse Mat & 0.0111 \\
\hline Wood (With Grain) & 0.0348 \\
\hline Corrugated Cardboard & 0.0585 \\
\hline Sandpaper & 0.0580 \\
\hline
\end{tabular}

\section{Table 1: Standard deviation of mean surface profiles.}

\section{DISCUSSION AND CONCLUSION}

A method has been presented considering the use of Fourier series as a method of describing physical surface characteristics. The results presented suggest that a bandlimited Fourier series provides an accurate representation of small-scale surface details. In order to be employed in a haptic simulation using the high frequency probe augmented PHANToM, the texture description must be mapped onto a 3D object. A texture is first mapped onto a simple planar surface by creating a matrix of Fourier coefficients from two vectors created using equation 2. The resultant 2D Fourier series is described by equation 3 .

$$
\begin{gathered}
f(x, y)=\sum_{m=6}^{50}\left[\sum_{m=6}^{50} a_{n, m} \cos \left(n \omega_{0} x\right)+b_{n . m} \sin \left(n \omega_{0} x\right)\right] \\
a_{n, m} \cos \left(m \omega_{0} y\right)+b_{n, m} \sin \left(m \omega_{0} y\right)
\end{gathered}
$$

Where $\mathrm{a}$ and $\mathrm{b}$ are matrices, given by the product of two vectors with elements defined by a Gaussian distribution of mean and standard deviation defined by the surface properties, as outlined above. Finally, the plane is then mapped onto the 3D haptic object's surface, which can be achieved using several methods. To avoid boundary anomalies, the generated space can be reversed as the probe crosses a $50 \mathrm{~mm}$ boundary.
The aim of such haptic texture simulation is a procedural technique by which any surface can be described by tuning of a small number of parameters. The Fourier series method presented above may provide a realistic approximation to the surface profile of textures, however, for a $50 \mathrm{~mm}$ by $0.1 \mathrm{~mm}$ area of material, 2000 values (500 real and imaginary component means, and the 500 corresponding standard deviations for both real and imaginary parts) are required to describe the Fourier series template! Clearly, this is unacceptable if a large variety of surfaces are to be simulated in the haptic environment. It may be possible to reduce the number of terms employed in the Fourier series representation and still evoke the desired perceptual response. Basdogan et al[12] noted that several different textures could be generated by modifying a small number of parameters in a Fourier series. Future work will explore the possibility of reducing the number of Fourier coefficients, and the corresponding effect on the users perception of surface identifying characteristics.

The simulated textures were replayed to human subjects using a standard PHANToM, and with a PHANToM augmented with the high frequency probe. In preliminary tests, subjects were shown one of three surfaces; mousemat, sanpaper or cardboard. While using the probe, subjects could regularly identify the correct surface, though when prompted for a qualitative assessment, often commented that the surface did not resemble its real life counterpart. This was usually due to a lack of frictional forces tangential to the surface. With the PHANToM only, surfaces were unanimously described as 'rougher', and the mouse mat was often mistaken for the sandpaper. Again, the subjects noted lack of forces tangential to the surface.

The envisaged system is a 'black-box' type system, which takes subjective magnitude estimates of various surface qualities as it's input parameters, and as its output produces a spectral description of the surface profile. As descriptors of the perceptual dimensions of surface texture remain elusive, the proposed solution is largely unobtainable at present! However, most research tends to agree on the fact that a subject can quantitatively assess a surface in terms of its roughness, which appears to be a continuous dimension $[18,19,20]$. Possibilities for expansion and future work in the area include psychophysical appraisal of textures displayed using this method, with a view to establishing a Cartesian perceptual space capable of describing the identifying characteristics of object surfaces.

\section{ACKNOWLEDGEMENTS}

This work is supported by EPSRC GR/L76112 "Determining Appropriate Haptic Cues for Virtual Reality and Teleoperation". The authors would also like to thank Mr. Michael Hilton at the Department of Cybernetics, for his technical assistance.

\section{REFERENCES}

1. J.P. Fritz, K.E. Barner, Stochastic Models for Haptic Texture, Proc. SPIE Int. Symp. Intelligent Systems and Advanced Manufacturing Vol. 3901, pp. 34-44, 1996.

2. D. Katz, The World of Touch, Hillsdale, NJ., 1925.

3. M. Hollins, R. Faldowski, S. Rao, F. Young, Perceptual Dimensions of Tactile Surface Texture: A Multidimensional Scaling Analysis, Perception and Psychophysics 54, pp. $697-$ 705, 1993. 
4. D.A. Kontarinis, R.D. Howe, Display of High Frequency Tactile Information to Teleoperators, SPIE Vol. 2057, pp.4050, 1993.

5. R.L. Klatzky, S.J. Lederman, Identifying Objects from a Haptic Glance, Perception and Psychophysics 57, pp. 11111123, 1995.

6. R.L. Klatzky, J.M. Loomis, S.J. Lederman, H.Wake, N. Fujita, Haptic Identification of Objects and Their Depictions, Perception and Psychophysics 54, pp. 170-178, 1993.

7. D.A. Kontarinis, J.S. Son, W. Peine, R.D. Howe, A Tactile Shape Senseing and Display System for Teleoperated Manipulation, Proc. 1995 IEEE Intl. Conf. Robotics and Automation, pp. 641-646, 1995.

8. M. Minsky, M. Ouh-Young, O. Steele, F.P. Brooks jr., M. Behensky, Feeling and Seeing: Issues in Force Display, Proc. Symp. Interactive 3D Graphics, pp. 235-243, 1990.

9. T.H. Massie, Initial Haptic Explorations With the PHANToM: Virtual Touch Through Point Interaction, Thesis, Massachusetts Institute of Technology, 1996.

10. M. Akamatsu, S. Sato, I. MacKenzie, Multi-Modal Mouse: A Mouse-Type Device with Tactile and Force Display, Presence 3, pp.73-80, 1994.

11. A.M. Okamura, J.T. Dennerlein, R.D. Howe, Vibration Feedback Models for Virtual Environments, Proc. 1998 IEEE Intl. Conf. Robotics Automation, Leuven, Belgium, pp.674679, 1998.

12. C. Basdogan, C. Ho, M.A. Srnivasan, A Ray-Based Haptic Rendering Technique for Displaying Shape and Texture of $3 D$ Objects in Virtual Environments, DSC-VOL 61, Proc. Of the ASME Dynamic Systems and Control Division, pp. 7784, 1997.

13. D.F. Green, J.K. Salisbury, Texture Sensing and Simulation Using the PHANToM: Towards Remote Sensing of Soil Properties, Proc. of the Second PHANToM Users Group Workshop, MIT AI Technical Report No. 1617, RLE Technical Report No. 618, 1997.

14. J. Siira, D.K. Pai, Haptic Rendering - A Stochastic Approach, Proc. 1996 IEEE Intl. Conf. Robotics and Automation, Minneapolis, Minnesota, pp. 557-562, 1996.

15. S.A. Wall, W.S. Harwin, Mechatronic Design of a High Frequency Probe for Haptic Interaction, To appear in Proc. $6^{\text {th }}$ International Conference on Mechatronics and Machine Vision in Practice, Ankara, Turkey, 1-3 September, 1999.

16. R.T. Verrillo, Effect of Contactor Area on Vibrotactile Threshold, Journal Acous. Soc. America 35, pp. 1962-1966, 1963.

17. S.J. Lederman, R.L. Klatzky, Feeling Through A Probe, DSC-VOL 64, Proc. of the ASME Dynamic Systems and Control Division, 1998.

18. M. Minsky, S.J. Lederman, Simulated Haptic Textures: Roughness, Proc. ASME Dynamic Systems and Control Division 58, pp. 421-426, 1996.

19. S.J. Lederman, M.M. Taylor, Fingertip Force, Surface Geometry, and the Perception of Roughness by Active Touch, Perception and Psychophysics 12, 1972.

20. K.O. Johnson, S.S. Hsiao, Evaluation of the Relative Roles of Slowly and Rapidly Adapting Fibers in Roughness Perception, Can. J. Physiol. Pharmacol. 72, 1994. 